\title{
稀土材料在多相催化中的应用研究进展概述
}

\author{
郭金秋 ${ }^{a}$ 杜亚平 $*, b \quad$ 张洪波 $*, a, b$ \\ ( ${ }^{a}$ 南开大学材料科学与工程学院 国家新材料研究院 天津 300350) \\ ( ${ }^{b}$ 南开大学 天津市稀土材料与应用重点实验室 天津 300350)
}

\begin{abstract}
摘要 稀土在中国储量丰富, 尤其在徐光宪等老一辈科学家不懈努力下开发了高效提纯稀土元素的有效方法, 为当今 的稀土性能应用研究打下了坚实的基础. 稀土包括钪、钇以及镧系金属在内的 17 种元素, 由于镧系元素最外层电子和 次外层电子的结构相似 ([Xe $\left.] 4 \mathrm{f}^{n-15} \mathrm{~d}^{0 \sim 16} \mathrm{~s}^{2}(n=1 \sim 15)\right)$, 尤其是其独特的 $4 \mathrm{f}$ 电子层结构, 使稀土元素具有化学性质稳定、 化学价态可变、配位形式多样、总体具有 Lewis 酸性等特点, 使其在催化领域具有广泛的应用. 但稀土元素电子层结 构与催化反应效果之间的关系尚未有明确的总结, 因此本文从稀土材料的电子结构特点出发, 综述了其在多相(热)催 化领域中作为载体、Lewis 酸(碱)中心以及催化助剂等方面的应用.
\end{abstract}

关键词 稀土材料; 载体; Lewis 酸(碱); 催化助剂

\section{A Brief Summary of Research Progress on the Application of Rare Earth Materials in Heterogeneous Catalysis}

\author{
Guo, Jinqiu $^{a} \quad$ Du, Yaping*, $\quad$ Zhang, Hongbo ${ }^{*, a, b}$ \\ $\left({ }^{a}\right.$ School of Materials Science and Engineering \& National Institute for Advanced Materials, Nankai University, \\ Tianjin 300350) \\ ( ${ }^{b}$ Tianjin Key Laboratory of Rare Earth Materials and Application, Nankai University, Tianjin 300350)
}

\begin{abstract}
Rare earth (RE) resources are in big amount in China, which can be effectively purified based on the strategies developed by Prof. Guangxian Xu et al. last century, which sets up solid fundamentals for applied research on rare earth materials nowadays. Rare earth elements, including scandium, yttrium and lanthanides, feature stable overall chemical properties, variable valence states and coordination form as well as special Lewis acidity due to the unique electron configuration in the outermost and secondary outer orbitals of the lanthanide elements ([Xe] $4 \mathrm{f}^{n-15} \mathrm{~d}^{0 \sim 16} \mathrm{~s}^{2}(n=1 \sim 15)$ ), especially on their $4 \mathrm{f}$ electron shell structure, having been extensively used in catalysis. However, the efficiency and selectivity to the desired products are always the major challenges due to the complexity of catalysis, in particular, the mechanism by which rare earth metals affect catalytic reactions through structural or electronic effects has not been clarified. Therefore, this mini-review summarizes the research progress on the application of rare earth materials in heterogeneous catalysis (specifically on thermal catalysis). Firstly, a brief summary of rare earth materials' structural properties is provided with emphasis on the unique distribution of the $4 \mathrm{f}$ electron. Afterward, the application of RE elements in thermal catalysis was discussed in detail. For example: (1) as a support to promote catalytic reaction, such as $\mathrm{CeO}_{2}$, which has variable chemical valence and can be used as an active support to participate in the redox reaction; (2) as moderate Lewis acid (base) center to catalyze the aldol condensation of acetaldehyde/ethanol mixture and effectively control the $\mathrm{C}-\mathrm{C}$ bond coupling; (3) as electronic and structural promoters to improve catalytic activity and stability. Hence, the structure-function relationship is illustrated in accordance with the studies of the rare earth materials as the supports, Lewis acid (base) active center and catalytic promoters, suggesting great potential of rare earth materials in catalysis.
\end{abstract}

Keywords rare earth material; support; Lewis acid (base); catalytic promoters

\section{1 引言}

钪、钎以及镧系(镧、铈、镨、钕、铝、钐、铕、 钝、铽、镝、钬、饵、铥、镱、镥)构成稀土元素. 自 18 世纪以来, 稀土元素在生物学、军事、农业以及化学
工程等领域具有非常广泛的应用 ${ }^{[1]}$. 我国稀土资源丰富, 种类齐全，且储存总量位居世界第一位，是世界上最大 的稀土生产、应用和出口国 ${ }^{[2]}$.

近年来, 基于稀土元素自身的特点, 如: 结构相对 稳定、具有中等强度的 Lewis 酸、具有独特的 $4 \mathrm{f}$ 电子层

*E-mail: ypdu@nankai.edu.cn; hbzhang@nankai.edu.cn

Received March 4, 2020; published June 22, 2020.

Project supported by the "111 Project" of China (No. B18030), Nankai University (No. 023-92022018), Open Foundation of Key Laboratory of Industrial Ecology and Environmental Engineering of Ministry of Education, Dalian University of Technology (No. KLIEEE-19-07) and Natural Science Foundation of Tianjin (No. BE122121).

项目受中国“111 项目”(No. B18030)、南开大学(No. 023-92022018)、大连理工大学工业生态与环境工程教育部重点实验室开放基金(No. KLIEEE-19-07) 以及天津市自然科学基金(No. BE122121)资助. 
结构, 使其在光催化 ${ }^{[3-6]}$ 、电催化 ${ }^{[7,8]}$ 、热催化 ${ }^{[9-11]}$ 领域具 有广泛的应用. 但由于催化反应的复杂性使得催化反应 产物分布广泛, 不能有效地合成目标产物. 究其主要原 因在于人们对于稀土元素通过结构、电子效应来影响催 化反应的机理尚不明确. 本综述依据稀土独特的电子层 排布, 选取典型实例 (集中讨论热催化), 概述了其作为 催化载体、Lewis 酸(碱)中心、催化助剂的应用, 并对稀 土材料的发展前景进行了展望.

\section{2 稀土材料概述}

\section{1 稀土元素的电子构型特点}

稀土金属具有独特的电子构型, 以镧系元素为例, 其电子构型为 $[\mathrm{Xe}] 4 \mathrm{f}^{n-1} 5 \mathrm{~d}^{0 \sim 1} 6 \mathrm{~s}^{2}(n=1 \sim 15)$, 最外层都填 充到 $6 \mathrm{~s}^{2}$ 轨道, $5 \mathrm{~d}$ 轨道无电子填充或仅有一个电子填充, 且容易失去外层两个 $6 \mathrm{~s}$ 轨道电子和一个次外层 $5 \mathrm{~d}$ 轨道 上的电子, 无 $\mathrm{d}$ 轨道电子时则失去一个 $4 \mathrm{f}$ 轨道电子, 从 而实现 $4 \mathrm{f}^{n} \rightarrow 4 \mathrm{f}^{n-1} 5 \mathrm{~d}^{1}$ 的转变, 使稀土离子通常表现为 +3 价. 根据洪特规则可知, 在原子的电子结构中, 当 同一层的原子处于全空、半满或全满时原子相对稳定, 对于稀土元素, 具有 $[\mathrm{Xe}] 4 \mathrm{f}^{0} 、[\mathrm{Xe}] 4 \mathrm{f}^{7}$ 和 $[\mathrm{Xe}] 4 \mathrm{f}^{14}$ 电子构 型的 $\mathrm{La}^{3+} 、 \mathrm{Gd}^{3+} 、 \mathrm{Lu}^{3+}$ 比较稳定, 而对于比它们原子序 数稍大的 $\mathrm{Ce}^{3+} 、 \mathrm{Pr}^{3+} 、 \mathrm{~Tb}^{3+}$, 比稳定态离子多 $1 \sim 2$ 个电 子, 所以易被氧化为 +4 价; 比它们原子序数小的 $\mathrm{Sm}^{3+} 、 \mathrm{Eu}^{3+} 、 \mathrm{Yb}^{3+}$ 离子, 比稳定态离子少 $1 \sim 2$ 个电子, 因此易被还原成 +2 价 ${ }^{[12]}$.

\section{2 稀土材料在催化领域的适用性}

部分稀土材料的化学价态可变, 可以将其应用于催 化氧化(还原)反应, 比如, $\mathrm{CeO}_{2}$ 中铈离子可以实现从 +3 价到 +4 价的可逆转变, 同时释放出氧空位, 从而使 $\mathrm{CeO}_{2}$ 具有独特的储存/释放氧的能力 ${ }^{[13-19]}$. 而 $\mathrm{La}$ 元素由 于 4f 轨道全空而十分稳定, 利用这一特性, 可以将 $\mathrm{La}_{2} \mathrm{O}_{3}$ 引入催化体系, 以提高催化材料的稳定性 ${ }^{[20,21]}$. 另外, 由于稀土元素存在大量的 $4 \mathrm{f}$ 空轨道, 使其在与其 他材料复合以及掺杂作为催化助剂时, 可以与载体产生 较强的相互作用, 从而表现出优良的催化效果 ${ }^{[22-35]}$.

\section{3 稀土材料在催化中的应用}

基于稀土元素独特的电子层结构, 稀土材料被广泛 地应用于各种催化转化, 包括光催化 ${ }^{[3-6]}$ 、电催化 ${ }^{[7,8]}$ 和热 催化 ${ }^{[9-11]}$, 本综述主要从稀土材料作为载体、Lewis 酸 (碱)催化剂, 以及作为催化助剂三个方面, 介绍稀土材 料用于(热)催化领域时表现出的特异性.

\section{1 作为载体}

\subsection{1 $\mathrm{CeO}_{2}$}

在催化反应中, 载体不仅影响负载金属粒子的形貌 和结构, 还会通过与金属粒子的相互作用来影响催化反
应活性、选择性和稳定性 ${ }^{[13]}$ ，因此选用合适的载体有助 于催化反应的顺利进行. 与 $\mathrm{SiO}_{2}$ 等惰性载体不同, 以 $\mathrm{CeO}_{2}$ 为典型代表的化学价态可变的部分稀土金属氧化 物为载体时, 可作为活性载体, 参与到氧化还原反应之 中. 例如, Mattos 等 ${ }^{[14]}$ 根据 $\mathrm{CeO}_{2}$ 可参与到氧化还原反应 中的特性, 在 $\mathrm{CeO}_{2}$ 载体上负载 $\mathrm{Pt}$, 将其用作乙醇选择 性氧化催化剂, 分析了乙醇转化机理. 发现其反应过程 明显有 $\mathrm{CeO}_{2}$ 参与，具体反应路径如下:

$$
\begin{gathered}
\mathrm{CH}_{3} \mathrm{CH}_{2} \mathrm{OH}(\mathrm{g})+\mathrm{Ce}^{4+} \rightarrow \mathrm{CH}_{3} \mathrm{CH}_{2} \mathrm{O}-\mathrm{Ce}^{4+}+(1 / 2) \mathrm{H}_{2} \\
\mathrm{CH}_{3} \mathrm{CH}_{2} \mathrm{O}-\mathrm{Ce}^{4+} \rightarrow \mathrm{CH}_{3} \mathrm{CHO}-\mathrm{Ce}^{4+}+(1 / 2) \mathrm{H}_{2}
\end{gathered}
$$

$\mathrm{CH}_{3} \mathrm{CHO}-\mathrm{Ce}^{4+}+\mathrm{Ce}^{4+}-\mathrm{O}^{2-} \rightarrow \mathrm{Ce}^{3+}-\mathrm{CH}_{3} \mathrm{COO}-\mathrm{Ce}^{3+}+$ $(1 / 2) \mathrm{H}_{2}$

当温度进一步升高时, 部分氧化的产物则会进一步 分解, 产生 $\mathrm{CH}_{4}, \mathrm{CO}$ 和 $\mathrm{H}_{2}$ 等副产物.

$\mathrm{CeO}_{2}$ 是商用汽车三效催化剂的载体, 三效催化剂 是用来催化转化汽油燃烧过程中产生的不完全燃烧的 碳氢化合物 $\mathrm{CH}_{x}$, 氮氧化合物 $\mathrm{NO}_{x}$ 和 $\mathrm{CO}$ 等对环境有害 的污染物 ${ }^{[15-17]}$. 由于无法控制汽车尾气中的氧含量, 当 氧含量不足时, 会导致尾气的不完全燃烧，从而使污染 物残留. 研究表明, 采用 $\mathrm{Rh} 、 \mathrm{Pt}$ 等贵金属和二氧化铈形 成的复合材料作为三效催化剂来储存氧气，可获得接近 化学计量比的污染物转化率, 但是当燃料十分充足或极 其匮乏时, 这种三效催化剂的催化效率会显著降低. 当 $\mathrm{CeO}_{2}$ 载体含量增加时, 催化剂储存/释放氧的能力也相 应提高, 因此增加 $\mathrm{CeO}_{2}$ 载体含量会显著改善这一不 足 ${ }^{[18]}$, 在燃料充足的条件下, $\mathrm{Ce}^{4+}$ 被还原成 $\mathrm{Ce}^{3+}$ ，同时 释放氧; 然而当缺少燃料时, 铈的化学价则从 +3 价转 变成 +4 价, 并储存氧. 利用 $\mathrm{CeO}_{2}$ 化学价态可变的特性, 提高了汽车三效催化剂的转化效率和抗老化能力 ${ }^{[19]}$. $\mathrm{CeO}_{2}$ 作为载体时, 可以通过改变 $\mathrm{CeO}_{2}$ 的形貌以获得不 同的催化性能 ${ }^{[36-39]}$. 贾春江等 ${ }^{[36]}$ 将质量分数为 $1 \%$ 的铜 分别沉积到 $\mathrm{CeO}_{2}$ 纳米立方体和纳米棒中, 用于催化 $\mathrm{CO}$ 氧化. 结果发现 $\mathrm{Cu}$ 可在暴露 $\{100\}$ 面的 $\mathrm{CeO}_{2}$ 纳米立方 体上产生 $\mathrm{Cu}(\mathrm{I})$ 活性位点, 这种活性位点可以在 $\mathrm{CO}$ 氧化 的过程中产生 $\mathrm{Cu}(\mathrm{I})-\mathrm{CO}$ 活性物种, 从而提高催化 $\mathrm{CO}$ 氧 化的能力. Flytzani-Stephanopoulos 等 ${ }^{[38,39]}$ 将 $\mathrm{Au}$ 负载在 $\mathrm{CeO}_{2}$ 上, 用于低温催化水煤气变换反应. 其采用两步 法, 分别将 $\mathrm{Au}$ 负载在 $\mathrm{CeO}_{2}$ 纳米棒、纳米立方体和纳米 多面体上, 得到了不同的水煤气变换反应活性. 透射电 镜(TEM)和高分辨透射电镜(HRTEM)表征表明(如图 1 所示) $\mathrm{Au}$ 在 $\mathrm{CeO}_{2}$ 纳米立方体上暴露 $\{111\}$ 面. 三种形貌 的 $\mathrm{Au} / \mathrm{CeO}_{2}$ 催化剂(图 2)的活性顺序为: $\mathrm{Au} / \mathrm{CeO}_{2}$ 纳米棒 $>\mathrm{Au} / \mathrm{CeO}_{2}$ 纳米多面体 $>\mathrm{Au} / \mathrm{CeO}_{2}$ 纳米立方体, 且在 $350{ }^{\circ} \mathrm{C}$ 的反应温度下, $\mathrm{Au} / \mathrm{CeO}_{2}$ 纳米棒的转化率接近 $100 \%$, 而 $\mathrm{Au} / \mathrm{CeO}_{2}$ 纳米立方体的转化率在 $350{ }^{\circ} \mathrm{C}$ 时只 有 $20 \%$. 这是由于: (1) $\mathrm{CeO}_{2}$ 的纳米尺寸效应, 这种纳米 尺寸效应可以提高 $\mathrm{CeO}_{2}$ 固有的还原性, 将 $\mathrm{Ce}^{4+}$ 还原成 
$\mathrm{Ce}^{3+}$, 同时产生氧空位 ${ }^{[40]}$; (2) $\mathrm{CeO}_{2}$ 的形貌会显著影响 $\mathrm{Au}$ 的活性, $\mathrm{CeO}_{2}$ 纳米棒暴露 $\{110\}$ 晶面, 氧空位在 $\{110\}$ 晶面上的生成能低于 $\{100\}$ 和 $\{111\}$ 晶面, 这些氧空位可 以稳定 $\mathrm{Au}$ 金属离子, 从而有利于提高 $\mathrm{Au} / \mathrm{CeO}_{2}$ 纳米棒 的活性; (3)其活性的提高也与晶格应力有关. 这一手段 同样可以拓展到研究其它氧化物形貌对催化活性的影 响.
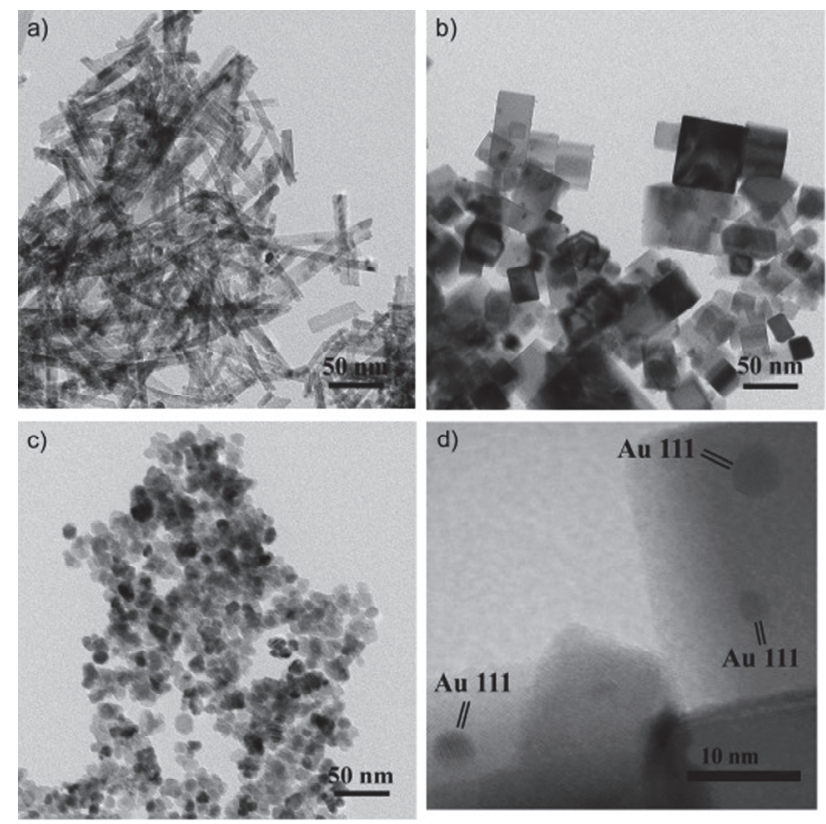

图 $11 \%$ 原子分数的 $\mathrm{Au}$ 负载在 $(\mathrm{a}) \mathrm{CeO}_{2}$ 纳米棒、 $(\mathrm{b}, \mathrm{d})$ 纳米立方体和 (c)纳米多面体的 TEM $(\mathrm{a} \sim \mathrm{c})$ 和 HRTEM (d) 图像 ${ }^{[38]}$

Figure 1 TEM $(\mathrm{a} \sim \mathrm{c})$ and HRTEM (d) images of 1 atom $\% \mathrm{Au}$ on (a) $\mathrm{CeO}_{2}$ nanorods, (b, d) nanocubes and (c) nanopolyhedra ${ }^{[38]}$

$\mathrm{CeO}_{2}$ 作为载体时, 还可与负载在其上的金属 $(\mathrm{Pd}$, $\mathrm{Ru}, \mathrm{Rh}$ 等)产生较强的相互作用(Strong Metal-Support Interaction, SMSI), 这种强相互作用可以提高一些电子 敏感型催化转化速率, 并可以有效地控制反应产物的选 择性, 如 $\mathrm{CeO}_{2}$ 为载体用于催化 $\mathrm{CO}$ 氧化 ${ }^{[41-47]}$ 、水煤气变 换 ${ }^{[48-50]}$ 和 $\mathrm{CO}_{2}$ 加氢 ${ }^{[51]}$ 等反应. 乔波涛等 ${ }^{[45]}$ 使用单原子金 和金纳米颗粒对 $\mathrm{CeO}_{2}$ 载体进行修饰, 并对比了其催化 $\mathrm{CO}$ 氧化的活性, 结果发现 $\mathrm{CeO}_{2}$ 和单原子金之间的相互 作用有利于形成氧化态的金, 在水存在的条件下可作为 电子受体, 为 $\mathrm{CO}+\mathrm{OH} \rightarrow \mathrm{COOH} \rightarrow \mathrm{CO}_{2}+\mathrm{H}$ 提供有效的 反应通道, 增强了催化 $\mathrm{CO}$ 氧化的反应活性. 通过金属 与载体之间的强相互作用(SMSI), 载体不仅可以影响负 载金属的分散性, 而且可以影响贵金属纳米颗粒的化学 状态 ${ }^{[52]}$. 例如: 马丁课题组 ${ }^{[53]}$ 用无配体的方法在 $\mathrm{CeO}_{2}$ 纳米片上合成了高度分散、Ir 含量不同的纳米颗粒, 并 将其用于 $\mathrm{CO}_{2}$ 加氢反应. 其研究发现, 可以通过改变负 载在 $\mathrm{CeO}_{2}$ 上 Ir 纳米颗粒的尺寸来改变产物的选择性. 图 3 显示不同 $\mathrm{Ir}$ 负载量的 $\mathrm{Ir} / \mathrm{Ce}$ 催化剂中, $\mathrm{CO}$ 和 $\mathrm{CH}_{4}$ 选 择性和 Ir-Ir 和 Ir-O 配位数(Coordination Number, CN)之 间的关系, 当 $\mathrm{Ir}$ 加入量从 $20 \%$ (质量分数)减少到 5\%(质 量分数)时, Ir 的尺寸降低, $\mathrm{CN}_{\text {Ir-Ir }}$ 从 9.7 降低为 7.2 , 但
$\mathrm{CN}_{\text {Ir-O }}$ 逐渐升高，这表明当 $\mathrm{Ir}$ 占比小时，氧原子会对 $\mathrm{Ir}$ 物种进行修饰, 这种结构修饰来源于 $\mathrm{CeO}_{2}$ 载体与 $\mathrm{Ir}$ 之 间的强相互作用，使更多的氧原子富集在金属 Ir 表面， 从而使 $\mathrm{CN}_{\mathrm{Ir}-\mathrm{O}}$ 升高. 部分氧化的 $\mathrm{Ir} / \mathrm{CeO}_{2}$ 有利于 $\mathrm{CO}_{2}$ 的还 原并促进 $\mathrm{CO}$ 的脱附，使 $\mathrm{CO}$ 的选择性提高，导致 $5 \%$ $\mathrm{Ir} / \mathrm{CeO}_{2}$ 上 $\mathrm{CO}$ 选择性接近 $100 \%$.

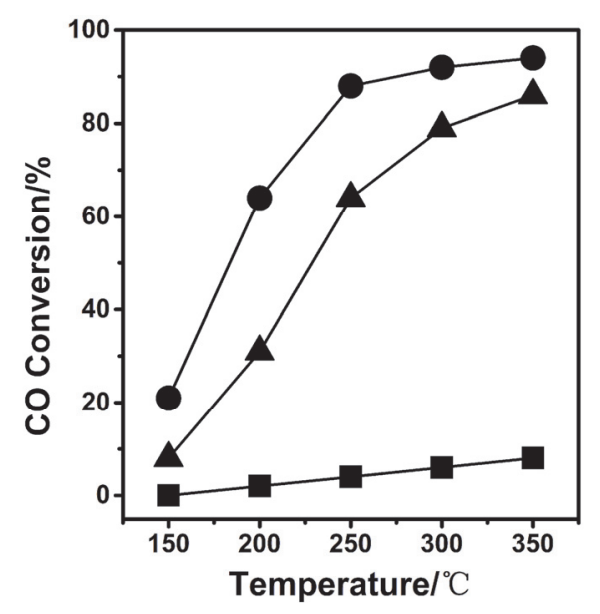

图 $21 \mathrm{atom} \% \mathrm{Au} / \mathrm{CeO}_{2}$ 纳米棒 $(\boldsymbol{O})$, 纳米立方体 $(\boldsymbol{\square})$, 纳米多面体 $(\boldsymbol{\Delta})$ 用于水煤气变换反应时 $\mathrm{CO}$ 转化率随温度的变化关系 ${ }^{[38]}$

Figure $2 \mathrm{CO}$ conversion as a function of temperature on water gas shift (WGS) reaction over 1 atom $\% \mathrm{Au}$ on $\mathrm{CeO}_{2}$ nanorods $(\bullet)$, nanocubes (ם) and nanopolyhedra $(\boldsymbol{\Delta})^{[38]}$

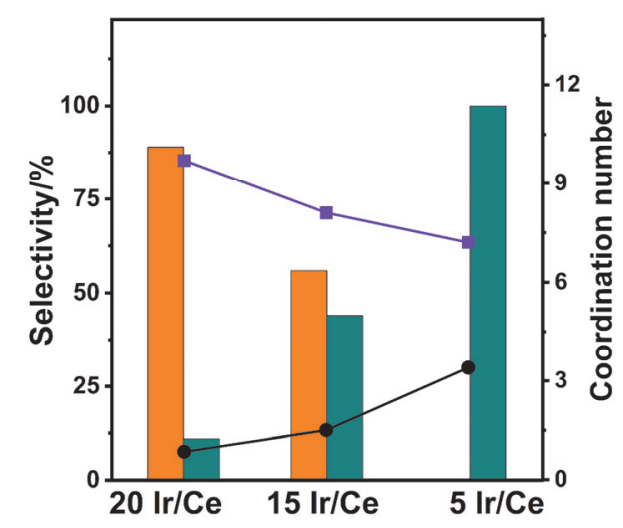

图 3 在不同 $\mathrm{Ir}$ 负载量的 $\mathrm{Ir} / \mathrm{Ce}$ 催化剂上, $\operatorname{Ir}-\operatorname{Ir}(\boldsymbol{\square})$ 和 $\mathrm{Ir}-\mathrm{O}(\boldsymbol{O})$ 配位数(数 据, 右轴)与催化选择性(棒, 左轴) (CO(橙色), $\mathrm{CH}_{4}$ (深青色))的关系 ${ }^{[33]}$ Figure 3 The coordination number $(\mathrm{CN})$ of Ir-Ir $(\mathbf{\square})$ and Ir-O (O) shells (data, right axis) correlate to catalytic selectivity (bars, left axis) (CO (orange), $\mathrm{CH}_{4}$ (dark cyan)) with $\mathrm{Ir} / \mathrm{Ce}$ catalysts at various Ir loadings $^{[53]}$

类似的，严纯华、张亚文等 ${ }^{[54}$ 近期通过改变 $\mathrm{Ru}$ 在 $\mathrm{Ru} / \mathrm{CeO}_{2}$ 中的尺寸(单原子、纳米团簇 $1.2 \mathrm{~nm}$ 、纳米颗粒 $4.0 \mathrm{~nm})$, 揭示了金属与载体之间的强相互作用随粒径 尺寸的变化规律，同时讨论了 $\mathrm{H}$ 溢流效应与水脱除反应 之间的竞争关系. 具体体现为: 当 $\mathrm{Ru}$ 是单原子时, $\mathrm{CeO}_{2}$ 与 $\mathrm{Ru}$ 之间的相互作用最强，可以抑制金属羰基的活化; 而当 $\mathrm{Ru}$ 是纳米颗粒时, $\mathrm{H}$ 溢流效应最强，能够抑制表面 水的脱除; 当 $\mathrm{Ru}$ 是纳米团簇时, 金属与载体间的相互 作用以及 $\mathrm{H}$ 溢流效应在 $\mathrm{Ru} / \mathrm{CeO}_{2}$ 纳米团簇催化剂上达到 平衡，因此该复合材料表现出最佳的低温 $\mathrm{CO}_{2}$ 甲烷化的 
活性，甲烷的选择性可达 $98 \% \sim 100 \%$.

\subsection{2 $\mathrm{RE}_{2} \mathrm{O}_{3}$}

稀土金属氧化物 $\mathrm{RE}_{2} \mathrm{O}_{3}$ (Rare Earth, $\mathrm{RE}$ ) 可作为催化 剂的载体 ${ }^{[55]}$, 以 $\mathrm{La}_{2} \mathrm{O}_{3}$ 作为典型代表, 来说明 $\mathrm{RE}_{2} \mathrm{O}_{3}$ 作 为载体的作用. 镧元素的 $4 \mathrm{f}$ 轨道含有 0 个电子, 相对稳 定, 因此可利用 $\mathrm{La}_{2} \mathrm{O}_{3}$ 作为载体来提高催化体系的稳定 性 ${ }^{[20,21]} . \mathrm{La}_{2} \mathrm{O}_{3}$ 作为载体时, 可以通过与金属粒子之间的 相互作用 ${ }^{[56,57]}$, 尤其是静电相互作用来促进金属粒子的 分散. Flytzani-Stephanopoulos 等 ${ }^{[58]}$ 用阴离子吸附法制备 了 $\mathrm{Au} / \mathrm{La}_{2} \mathrm{O}_{3}$ 催化剂, $\mathrm{Au}$ 前驱体和镧基载体界面之间具 有静电相互作用, 这种静电相互作用可在不降低 $\mathrm{Au}$ 担 载量的前提下, 使 $\mathrm{Au}$ 高度分散在 $\mathrm{La}_{2} \mathrm{O}_{3}$ 载体中. 这种独 特的静电相互作用还可以消除体系中残留的氯离子, 有 利于在低温下进行水煤气转化, 转化率可达 $70 \% . \mathrm{La}_{2} \mathrm{O}_{3}$ 还可以作为活化烷烃制低碳烯烃的载体. 烷烃裂解制备 低碳烯烃时, $\mathrm{C}-\mathrm{H}$ 键的活化往往是反应的决速步 ${ }^{[59]}$, 选用高活性的活化 $\mathrm{C}-\mathrm{H}$ 键的材料作为催化剂对整体反 应有利. 但是 $\mathrm{C}-\mathrm{H}$ 键的活化能垒较高, 从而导致反应 体系能耗相对较高, 且产物分布多样. 根据这一问题, Hardacre 等 ${ }^{[60]}$ 对不同氧化物载体制备烯烃的效果进行 了测试, 催化性能如表 1 所示, 其中 $\mathrm{La}_{2} \mathrm{O}_{3}$ 展现了相对 较高的总体反应速率 $\left(0.84 \mathrm{mmol} \cdot \mathrm{min}^{-1} \cdot \mathrm{g}^{-1}\right)$ 及最高的烯

表 1 在 $650{ }^{\circ} \mathrm{C}$ 下氧化物催化丁烷裂解的催化结果 ${ }^{[60]}$

Table 1 Catalytic performance on the oxidative cracking of $n$-butane at $650{ }^{\circ} \mathrm{C}^{[60]}$

\begin{tabular}{llllllll}
\hline \multirow{2}{*}{ 催化剂 } & $\begin{array}{l}\text { 反应速率/(mmol・ } \\
\left.\min ^{-1} \cdot \mathrm{g}^{-1}\right)\end{array}$ & \multicolumn{3}{c}{ 选择性 $/ \%$} & \multicolumn{2}{c}{ 产率/\% } \\
\cline { 4 - 7 } & 烯烃 & 烷烃 & $\mathrm{CO}_{x}$ & $\mathrm{C}_{2} \mathrm{H}_{4}$ & $\mathrm{C}_{3} \mathrm{H}_{6}$ \\
\hline $\mathrm{La}_{2} \mathrm{O}_{3}$ & 0.84 & 28.8 & 3.2 & 23.8 & 1.7 & 1.7 \\
$\mathrm{CeZrO}_{4}$ & 0.86 & 17.9 & 2.2 & 69.5 & 1 & 0.3 \\
$\mathrm{ZrO}_{2}$ & 0.38 & 16.3 & 3.8 & 24.5 & 0.4 & 0.5 \\
$\mathrm{TiO}_{2}$ & 0.51 & 17.3 & 2.1 & 26.3 & 1 & 0.3 \\
$\mathrm{Nb}_{2} \mathrm{O}_{5}$ & 0.25 & 20.5 & 5.3 & 27.2 & 0.2 & 0.3 \\
\hline
\end{tabular}

烃选择性(28.8\%). 之后将 $\mathrm{Au}$ 负载在 $\mathrm{La}_{2} \mathrm{O}_{3}$ 载体上, 并 将其用于丁烷裂解制烯烃中, 结果发现 $\mathrm{Au} / \mathrm{La}_{2} \mathrm{O}_{3}$ 复合 材料的催化活性优于单相材料, 且在反应 $48 \mathrm{~h}$ 之后仍可 保持较高活性(转化率稳定在 38\%，烯烃选择性为 $62.5 \%$ 左右).

这是由于 $\mathrm{La}_{2} \mathrm{O}_{3}$ 有利于稳定离子态的金 $\left(\mathrm{Au}^{+}\right)$, 而不 是将金还原成零价 $\left(\mathrm{Au}^{0}\right), \mathrm{Au}^{+}$与 $\mathrm{La}_{2} \mathrm{O}_{3}$ 之间的相互作用 可以促进 $\mathrm{C}-\mathrm{H}$ 键的解离产生有效自由基，而未提供氧 化的位点, 使碳氧化合物的选择性降低, 碳氢化合物的 选择性升高. 龚学庆等 ${ }^{[61]}$ 和常春然等 ${ }^{[62]}$ 用密度泛函理 论(Density Functional Theory, DFT)对稀土金属氧化物活 化甲烷过程中 $\mathrm{C}-\mathrm{H}$ 键的解离进行了研究, 其中龚学庆 等 ${ }^{[61]}$ 发现调节 $\mathrm{La}_{2} \mathrm{O}_{3}$ 暴露的晶面或在 $\mathrm{La}_{2} \mathrm{O}_{3}$ 基体中掺杂 $\mathrm{Sr} / \mathrm{Ce}$ 离子 $\left(\mathrm{Sr} / \mathrm{Ce}-\mathrm{La}_{2} \mathrm{O}_{3}\right)$ 均可以促进 $\mathrm{C}-\mathrm{H}$ 键的解离. 其 通过 DFT 理论计算进行分析, 发现 $\mathrm{La}_{2} \mathrm{O}_{3}$ 中高密勒指数 的(210)晶面可暴露出低配位数的氧(尤其是 $\mathrm{O}_{2 \mathrm{c}}$ ), $\mathrm{Sr} / \mathrm{Ce}-$ $\mathrm{La}_{2} \mathrm{O}_{3}$ 可提供 $\mathrm{Ce} 4 \mathrm{f}$ 空轨道, 这两种方法均使 $\mathrm{La}_{2} \mathrm{O}_{3}$ 基材 料具有高的 $\mathrm{H}$ 吸附能、低的氧空位形成能，从而有效解 离甲烷形成甲基中间物种，提高碳氢化合物的选择性.

\subsection{Lewis 酸(碱)催化}

Lewis 酸、碱往往成对出现，对于不同的催化反应， 起作用的形式各异. 稀土元素具有独特的 Lewis 酸性, 与之结合的阴离子具有 Lewis 碱性, 因此可以将稀土材 料用做 Lewis 酸(碱)催化的催化剂.

近年来，由于化石能源储量日益降低，使能源短缺 问题更加凸显，人们发现该能源危机有望通过生物质平 台小分子催化转化为高附加值的产物来解决. 生物质乙 醇可以通过 ABE (Acetone-Butanol-Ethanol)发酵而大量 获得，且乙醇作为典型的生物质平台小分子，可以通过 aldol 加成反应生成异丁烯 ${ }^{[63]}$ 、丁醇 ${ }^{[64]}$ 、巴豆醛 ${ }^{[65]}$ 等高 附加值的化学品, 乙醛/乙醇混合物 aldol 加成的反应路 径如图式 1 .
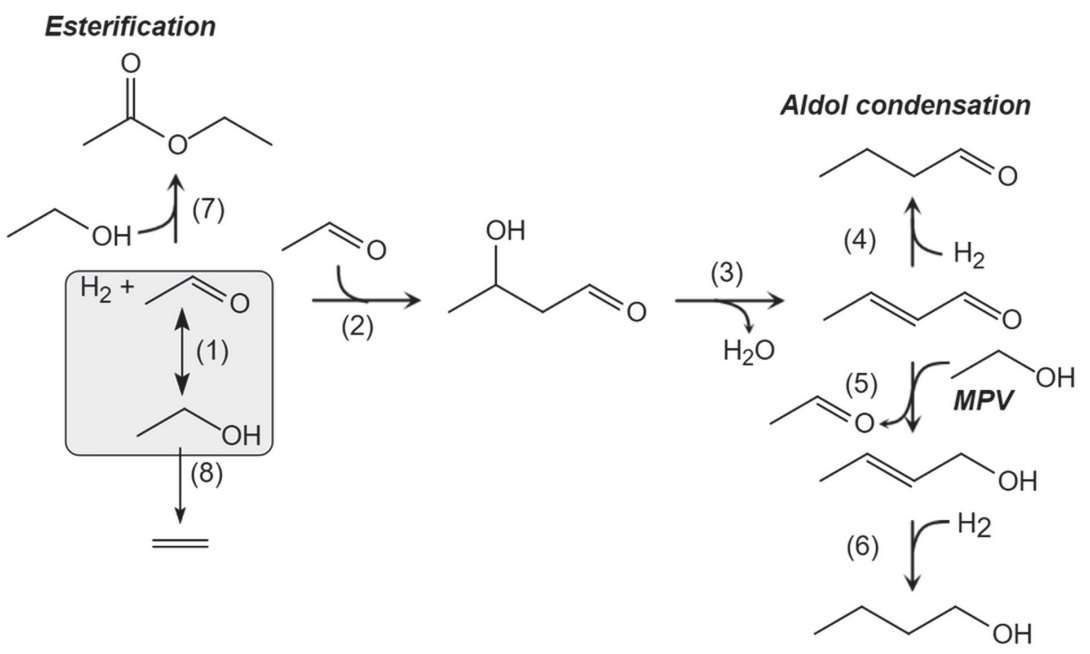

图式 1 乙醛/乙醇混合物 aldol 加成的反应路径

Scheme 1 Reaction network of aldol condensation from acetaldehyde/ethanol mixtures 
(1)乙醇脱氢生成乙醛; (2)两分子乙醛经过 aldol 加 成生成 aldol 产物一一-3-羟基丁醛; (3)3-羟基丁醛脱水生 成巴豆醛; (4)巴豆醛的碳碳键加氢生成丁醛; (5)巴豆醛 经过氢转移反应(Meerwein-Ponndorf-Verley, MPV 反应), 使碳氧键加氢生成巴豆醇; (6)巴豆醇进一步加氢生成丁 醇. 在反应过程中通常伴随着许多副反应的发生, 如(7) 乙醇与乙醛脱水生成乙酸乙酯、(8)乙醇直接脱水生成乙 烯等, 而且可能会进一步生成 $\mathrm{C}-\mathrm{C}$ 键从而得到高碳的 产物 ${ }^{[66]}$. 其中, $\mathrm{C}-\mathrm{C}$ 键的生成是 aldol 加成反应中的关 键步骤 ${ }^{67]}$, 然而, 经过一次 $\mathrm{C}-\mathrm{C}$ 键生成所获得的一级 aldol 产物之间会发生复杂的 $\mathrm{C}-\mathrm{C}$ 键交联反应, 使目标 产物的选择性不受控制. 大量的研究表明调控材料的 Lewis 酸(碱)强度可以显著影响 $\mathrm{C}-\mathrm{C}$ 键的生成 ${ }^{[68]}$. 稀土 元素具有独特的 Lewis 酸性, 且构成稀土金属氧化物时 存在 Lewis 酸、碱对 ${ }^{[69]}$ (稀土元素作为 Lewis 酸, 氧原子 作为 Lewis 碱), 因此可以利用稀土材料独特的 Lewis 酸 (碱)特性, 用来催化 aldol 加成反应.

例如李兰冬等 ${ }^{[70]}$ 借助稀土独特的 Lewis 酸性构筑了 高效的 aldol 加成催化剂. 该课题组利用金属锌 $(\mathrm{Zn})$ 和稀 土元素钇 $(Y)$ 对 Beta 分子篮进行共修饰, 并将其作为乙 醇 aldol 加成反应的催化剂, 高选择性地制备了 1,3-丁二 烯(选择性约 $60 \%$ ). 并通过 ${ }^{1} \mathrm{H}$ 和 ${ }^{13} \mathrm{C}$ 的核磁共振波谱, 对 [Si]Beta, Zn/Beta, Y/Beta 以及 Zn-Y/Beta 进行表征. 结 果表明, $\mathrm{Y}$ 和 $\mathrm{Zn}$ 的加入可以在 Beta 分子篮中引入 Lewis 酸性位点, 使催化剂 aldol加成活性提高. 采用不同金属 修饰的 Beta 分子篎具有不同的催化反应活性及对应产 物选择性. 例如: $\mathrm{Zn} /$ Beta 的脱氢活性相对较高, 而 Y/Beta 具有较高的 aldol 加成反应活性. 为了进一步证 明 $\mathrm{Y}$ 是 aldol 加成反应的主要活性位点, 其在反应体系 中分别加入乙醇、巴豆醛以及乙醇与巴豆醛的混合物, 并用质谱在线检测不同催化剂所表现出的反应情况, 结 果如图 4 所示. 分析表明, aldol 加成反应主要在具有高 aldol 加成反应活性的 $\mathrm{Y}$ 活性位点上进行, 而 $\mathrm{Zn}$ 主要参 与乙醇脱氢生成乙醛, 进而进行 aldol 加成反应.
Wang 等 ${ }^{[71]}$ 的研究发现, 由于稀土元素有较低的电 负性，当用具有 Lewis 碱性的稀土金属氧化物 $\left(\mathrm{La}_{2} \mathrm{O}_{3}\right.$, $\mathrm{Y}_{2} \mathrm{O}_{3}$ ) 对镁铝水滑石进行修饰时, 可以减小水滑石的晶 粒尺寸、增大比表面积. 未加修饰、稀土修饰以及加水 修饰的镁铝水滑石的结构模型如图式 2 表示. 位于晶体 棱边和转角处的氧(低配位的氧)的 Lewis 碱性强于其他 位置处氧的碱性，当水滑石的晶粒尺寸减小时，低配位 氧数量增加, 从而使水滑石的碱性增强 ${ }^{[72,73]}$. 将稀土金 属 Y、La 修饰后的镁铝水滑石用作催化丙酮的 aldol 缩 合反应，表现出了很好的 aldol 加成活性. 如: 当用水对 稀土修饰的水滑石材料进一步修饰之后，在 $273 \mathrm{~K}$ 的反 应温度下，丙酮大多转化为亚异丙基丙酮，丙酮的转化 率可达 $20 \%$, 接近反应的平衡转化( $23.1 \%)$.

李小年等 ${ }^{[74]}$ 研究发现, 具有 Lewis 酸、碱对的二氧 化铈可以用于乙醛的 aldol 加成反应，其中稀土金属铈 作为路易斯酸中心, 晶格氧作为路易斯碱中心. 其根据 氧空位浓度的不同合成了四种 $\mathrm{CeO}_{2}$, 并对其 aldol 加成 效果进行了测定, 测试结果如图 5 所示. 从图中可以看 出, 随着 $\mathrm{CeO}_{2}$ 氧空位浓度的增大, aldol 加成产物的产率 也逐渐升高, 基本成线性增加的趋势，其中在最高的氧 空位浓度(43\%)下, aldol 加成产物的产率可达 65.7\%，巴 豆醛选择性高达 $88.3 \%$. 这是由于氧空位浓度最大的 $\mathrm{CeO}_{2}$, 在表面形成大量的氧空位, 增加了材料总体的 Lewis 碱性，从而有效催化乙醛的 aldol 加成反应. 这一 发现为设计高效的 aldol 加成反应催化剂提供了新思路.

\section{3 助剂}

催化助剂是指与催化活性组分产生特定作用从而 使得催化剂活性、选择性和稳定性得到明显改善的一类 物质. 根据助剂所起的不同作用，又可以将其分为防止 活性组分烧结、长大的结构型助剂和可以改变催化剂表 观电子态的电子性助剂 ${ }^{[75]}$. 由于稀土元素具有独特的 外层电子分布特性, 其 4f 轨道未完全充满, 有多个能 级、多个亚稳态, 可改变催化剂的酸碱性、活性组分的

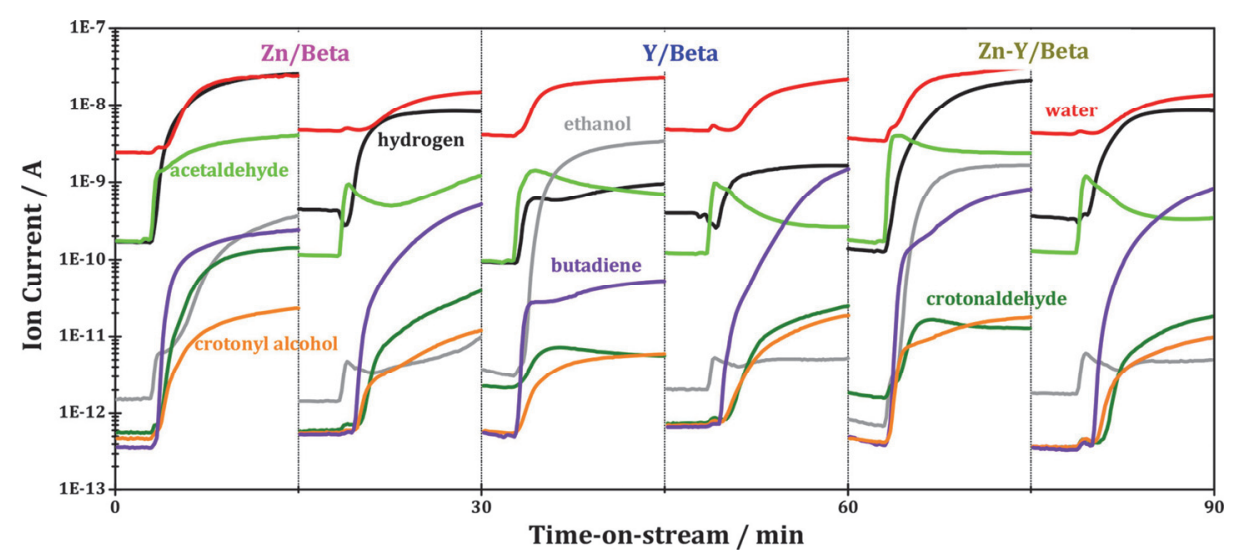

图 4 在 Zn/Beta、Y/Beta、Zn-Y-Beta 催化剂上, 分别用乙醇(左)、乙醇和巴豆醛(右)作为反应物的催化结果. 反应条件: 重量时空速度(WHSV) $=1 \mathrm{~h}^{-1}$, 反应温度 $T=623 \mathrm{~K}^{[70]}$

Figure 4 Modulation experiments about crotonaldehyde and ethanol over Zn/Beta, Y/Beta and Zn-Y-Beta catalysts (left: ethanol; right: ethanol and crotonaldehyde cofeeding). Reaction conditions: weight hourly space velocity (WHSV) $=1.0 \mathrm{~h}^{-1}$, reaction temperature of $T=623 \mathrm{~K}^{[70]}$ 


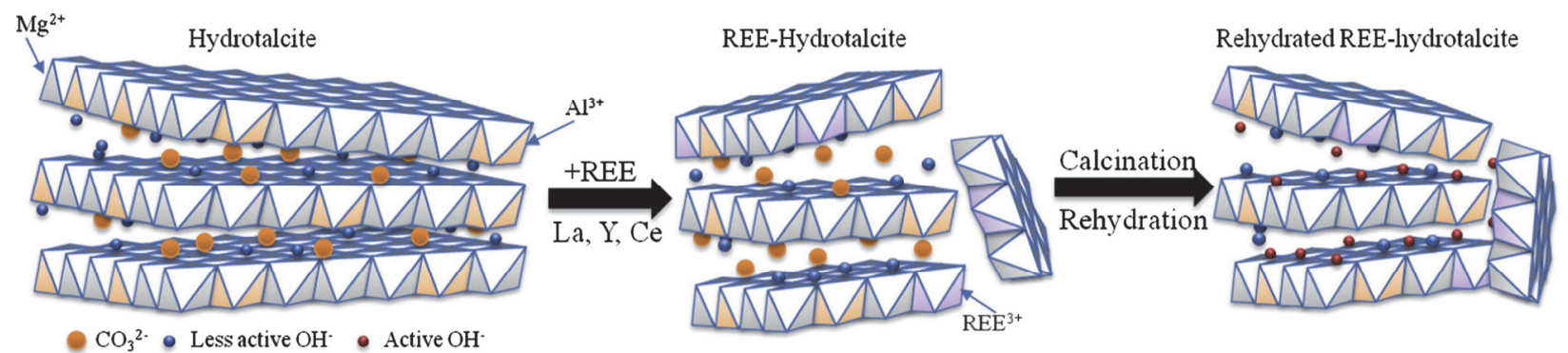

图式 2 未加修饰的 $\mathrm{MgAl}-\mathrm{HT}$ 、稀土( $\mathrm{La}, \mathrm{Y}, \mathrm{Ce})$ 修饰的 MgAl-HT 以及加水修饰的水滑石的结构模型[71]

Scheme 2 Schematic illustration of phase transition from unmodified MgAl-HT to rare earth (La, Y, Ce) modified MgAl-HT and modified hydrotalcite after rehydration ${ }^{[71]}$

电子态等诸多特性, 因此可以作为电子促进剂, 对催化 剂的电子结构进行调控 ${ }^{[76]}$. 同时, 还可以将稳定性优良 的稀土元素引入反应体系充当结构助剂 ${ }^{[77]}$, 对载体的 结构和形貌进行调控, 起到增加体系稳定性的作 用 ${ }^{[22-24]}$. 例如, Cunha 等 ${ }^{[76]}$ 将 $\mathrm{La}_{2} \mathrm{O}_{3}$ 引入 $\mathrm{Fe} / \mathrm{Al}$ 合金, 使 $\mathrm{La}_{2} \mathrm{O}_{3}$ 同时作为电子性助剂和结构助剂. 其采用原位热 处理法制备了 $\mathrm{Fe} / \mathrm{Al}$ 合金同 $\mathrm{La}_{2} \mathrm{O}_{3}$ 的混合物. 从 $\mathrm{X}$ 射线 光电子能谱(XPS)表征可以看出, $\mathrm{La}_{2} \mathrm{O}_{3}$ 的加入使得催化 剂中 $\mathrm{Fe} 2 \mathrm{p}_{3 / 2}$ 的结合能降低, 这说明 $\mathrm{La}_{2} \mathrm{O}_{3}$ 可充当电子性 助剂, $\mathrm{La}_{2} \mathrm{O}_{3}$ 的电子转移到了 $\mathrm{Fe}$ 上.

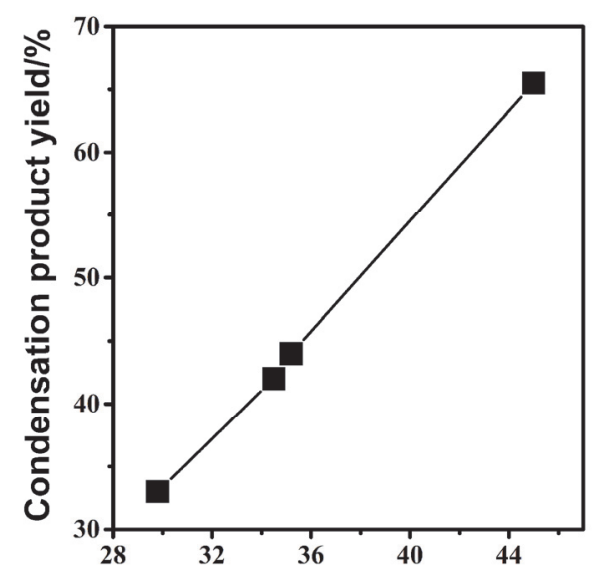

The concentration of oxygen vacancies $/ \%$

图 5 不同氧空位浓度的 $\mathrm{CeO}_{2}$ 催化剂中, 乙醇 aldol 缩合产物产率与 $\mathrm{CeO}_{2}$ 氧空位浓度的线性关系( ()$^{[74]}$

Figure 5 The linear correlation between the yields of ethanol condensation and the concentration of oxygen vacancies based on various $\mathrm{CeO}_{2}$ catalysts (- $)^{[74]}$

另外, 从表 2 可以看出, 加入适量的 $\mathrm{La}_{2} \mathrm{O}_{3}$ 可以避 免 $\mathrm{Fe} / \mathrm{Al}$ 合金的烧结, 从而增加该复合催化剂的比表面 积, 提高复合材料催化分解甲烷的活性和稳定性. 如图 6 所示, 在 $\mathrm{Fe} / \mathrm{Al}$ 为 $50 / 50$ 的合金中, 当 $\mathrm{La}_{2} \mathrm{O}_{3}$ 的质量分 数为 $20 \%$ 时, 催化甲烷分解的活性最好. 当 $\mathrm{La}_{2} \mathrm{O}_{3}$ 的质 量分数增加到 $50 \%$ 时, 催化性能反而有所降低. 这是由 于, $\mathrm{La}_{2} \mathrm{O}_{3}$ 加入量过多, 阻碍了活性位点的迁移, 从而导 致催化反应活性有所降低.
表 2 未加修饰、用 $20 \% \mathrm{La}_{2} \mathrm{O}_{3}$ 修饰的 $\mathrm{Fe} / \mathrm{Al}$ 合金催化剂的平均颗粒 尺寸和比表面积 ${ }^{[76]}$

Table 2 Average particle sizes $(d)$ and BET surface areas $\left(S_{\mathrm{BET}}\right)$ of $20 \%$ $\mathrm{La}_{2} \mathrm{O}_{3}$ modified and unmodified $\mathrm{Fe} / \mathrm{Al}$ alloy catalysts

\begin{tabular}{lll}
\hline 催化剂 & 平均颗粒尺寸 $d / \mu \mathrm{m}$ & $S_{\mathrm{BET}} /\left(\mathrm{m}^{2} \cdot \mathrm{g}^{-1}\right)$ \\
\hline $50 \mathrm{Fe}$ & 13 & 5 \\
$50 \mathrm{FeLa}(20)$ & 13 & 11 \\
$35 \mathrm{Fe}$ & 29 & 6 \\
$35 \mathrm{FeLa}(20)$ & 12 & 11 \\
\hline
\end{tabular}

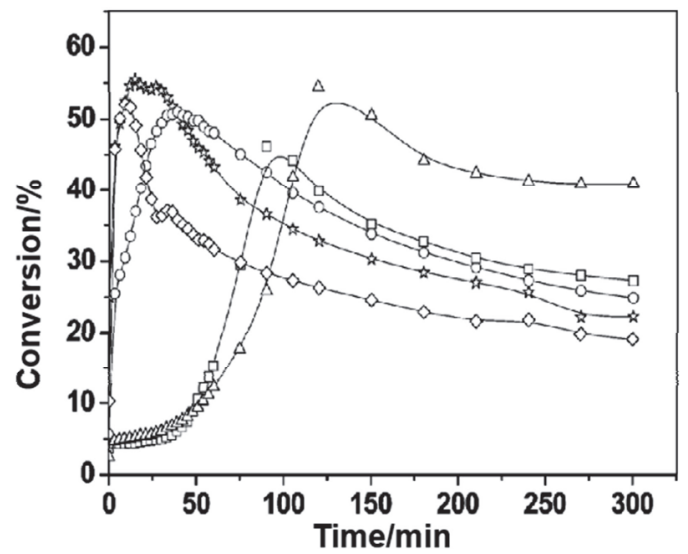

图 $6 \mathrm{La}_{2} \mathrm{O}_{3}$ 修饰的不同 $\mathrm{Fe} / \mathrm{Al}$ 合金催化剂 $(50 \mathrm{Fe}($ 约), $50 \mathrm{FeLa}(5)(\mathrm{\circ})$, $50 \mathrm{FeLa}(10)(\square), 50 \mathrm{FeLa}(20)(\Delta), 50 \mathrm{FeLa}(50)(\diamond))$ 催化甲烷分解反应活 性随时间变化曲线 ${ }^{[76]}$

Figure 6 Methane conversion comparison on $\mathrm{La}_{2} \mathrm{O}_{3}$ modified $\mathrm{Fe} / \mathrm{Al}$ alloy catalysts $(50 \mathrm{Fe}($ 行), $50 \mathrm{FeLa}(5)(0), 50 \mathrm{FeLa}(10)(\square), 50 \mathrm{FeLa}(20)(\Delta)$ and $50 \mathrm{FeLa}(50)(\diamond))^{[76]}$

Lercher 团队 ${ }^{[25]}$ 研究了在 $3 \%$ (质量分数)的 $\mathrm{La}$ 修饰的 $\mathrm{CeO}_{2}-\mathrm{ZrO}_{2}$ 上负载 $0.5 \%$ (质量分数)的 $\mathrm{Rh}$, 利用该催化剂 实现了乙酸向富氢气体的高效转化(重整反应), $\mathrm{H}_{2}$ 的选 择性高达 $98 \%$. 反应过程如图式 3.

其研究发现在 $\mathrm{CeO}_{2}-\mathrm{ZrO}_{2}$ 上负载 $\mathrm{Rh}$ 可以有效催化 乙酸的重整，但由于催化剂会烧结，在反应 $15 \mathrm{~h}$ 后会有 $20 \%$ 的失活. 为了解决催化剂烧结的问题, Lercher 等 ${ }^{[25]}$ 在催化剂中加入了少量 $\mathrm{La}_{2} \mathrm{O}_{3}$. 从图 7 中可以看出, 反应 $15 \mathrm{~h}$ 后, $\mathrm{Rh} / \mathrm{La}_{2} \mathrm{O}_{3} / \mathrm{CeO}_{2}-\mathrm{ZrO}_{2}$ 催化剂的反应活性几乎没 


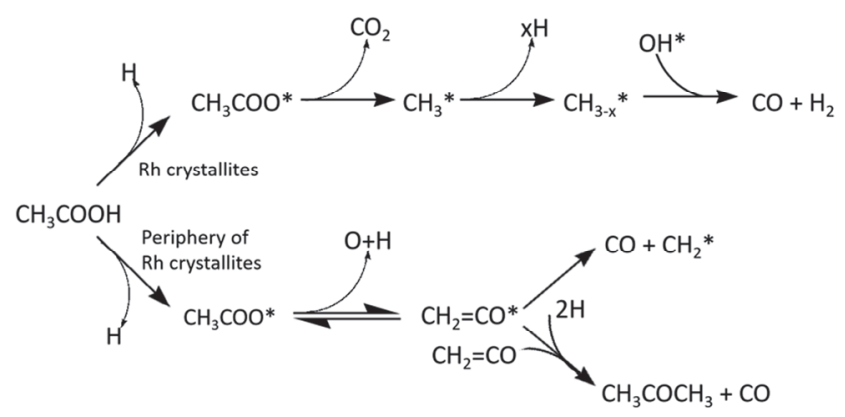

图式 3 在 $\mathrm{Rh} / \mathrm{La}_{2} \mathrm{O}_{3} / \mathrm{CeO}_{2}-\mathrm{ZrO}_{2}$ 催化剂上乙酸重整的反应路径图 ${ }^{[25]}$ Scheme 3 Reaction pathways of acetic acid reforming over $\mathrm{Rh} / \mathrm{La}_{2} \mathrm{O}_{3}$ / $\mathrm{CeO}_{2}-\mathrm{ZrO}_{2}$ catalyst $^{[25]}$

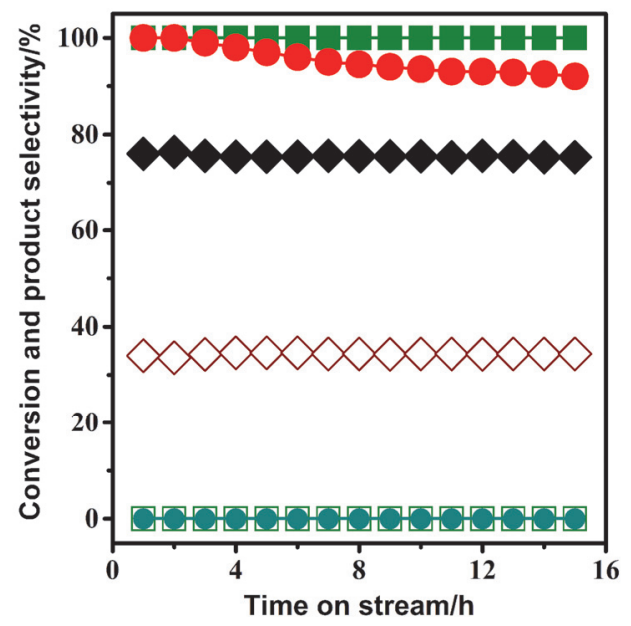

图 7 在 $\mathrm{Rh} / \mathrm{La}_{2} \mathrm{O}_{3} / \mathrm{CeO}_{2}-\mathrm{ZrO}_{2}$ 催化剂上的乙酸水蒸气重整, 反应时间 与转化率(红色 -)、产物选择性之间的关系 $\left(\mathrm{H}_{2}\right.$ (橄榄绿 $\left.\mathbf{-}\right) ; \mathrm{CO}_{2}$ (黑色 $\diamond) ; \mathrm{CO}$ (酒红色 $\diamond) ;\left(\mathrm{CH}_{3}\right)_{2} \mathrm{CO}$ (橄榄绿 口); $\mathrm{CH}_{4}$ (深青色 •)), 反应条 件: $T=650{ }^{\circ} \mathrm{C}, \mathrm{S} / \mathrm{C}=3$, 体积时空速度 $(\mathrm{GHSV})=28000 \mathrm{~h}^{-1[25]}$

Figure 7 Steam reforming of acetic acid over $\mathrm{Rh} / \mathrm{La}_{2} \mathrm{O}_{3} / \mathrm{CeO}_{2}-\mathrm{ZrO}_{2}$ catalyst. The conversion (red $\bullet$ ) and product selectivities $\left(\mathrm{H}_{2}\right.$ (olive $\left.\boldsymbol{\bullet}\right)$, $\mathrm{CO}_{2}$ (black $\left.\diamond\right), \mathrm{CO}$ (wine $\left.\diamond\right),\left(\mathrm{CH}_{3}\right)_{2} \mathrm{CO}$ (olive $\left.\square\right), \mathrm{CH}_{4}($ dark cyan $\bullet$ )) as a function of time $\left(T=650{ }^{\circ} \mathrm{C}, \mathrm{S} / \mathrm{C}=3\right.$, gas hourly space velocity $\left.(\mathrm{GHSV})=28000 \mathrm{~h}^{-1}\right)^{[25]}$

有下降, 这是因为 $\mathrm{La}_{2} \mathrm{O}_{3}$ 的加入提高了晶格氧的迁移速 率, 从而限制了积炭前驱体的浓度, 避免了大量积碳的 产生, 增加了载体的稳定性, 有效地抑制了催化剂的失 活，使催化剂的稳定性提高.

镍在甲烷重整过程中有利于 $\mathrm{CO}_{2}$ 的活化, 且价格相 对便宜, 因此广泛用于甲烷干法重整反应 ${ }^{[26-29]}\left(\mathrm{CH}_{4}+\right.$ $\mathrm{CO}_{2} \rightarrow 2 \mathrm{CO}+2 \mathrm{H}_{2}$ ), 但由于镍表面容易积碳, 导致其很 容易失活, 因此需要找到合适的助催化剂来抑制表面积 碳的产生 ${ }^{[30]}$. 大多数稀土金属和氧化物都可以用作镍 基催化剂的助剂, 钝、铈和镧等稀土元素被广泛用于修 饰镍基催化剂 ${ }^{[31-33]}$, 其中借助镧氧化物来修饰镍基催化 剂最为常见 ${ }^{[32-34]}$. 综合之前的科研成果, 武志坚等 ${ }^{[35]}$ 采 用 $\mathrm{La}$ 或者 $\mathrm{La}_{2} \mathrm{O}_{3}$ 对镍的(111) 晶面进行修饰, 很可能改变 了整体的反应路径，在两种材料上不同反应路径的反应 速率在图 8 中表示. 可以看出在 $\mathrm{La} / \mathrm{Ni}(111)$ 表面, 反应被 证实以 $\mathrm{P} 2$ 反应 $\left(\mathrm{CH}_{4}+\mathrm{CO}_{2} \rightarrow \mathrm{CH}+3 \mathrm{H}+\mathrm{CO}+\mathrm{O} \rightarrow \mathrm{CHO}\right.$ $\left.+3 \mathrm{H}+\mathrm{CO} \rightarrow 2 \mathrm{CO}+2 \mathrm{H}_{2}\right)$ 为主, 而在 $\mathrm{La}_{2} \mathrm{O}_{3} / \mathrm{Ni}(111)$ 表面, 则以 $\mathrm{P} 4$ 反应 $\left(\mathrm{CH}_{4}+\mathrm{CO}_{2}+\mathrm{La}_{2} \mathrm{O}_{3} \rightarrow \mathrm{CH}+\mathrm{CO}_{2}\left(\mathrm{La}_{2} \mathrm{O}_{3}-\mathrm{O}\right)\right.$ $\left.+3 \mathrm{H} \rightarrow 2 \mathrm{CO}+2 \mathrm{H}_{2}+\mathrm{La}_{2} \mathrm{O}_{3}\right)$ 为主. $\mathrm{La}_{2} \mathrm{O}_{3} / \mathrm{Ni}(111)$ 在高温下 的催化活性和稳定性优于 $\mathrm{La} / \mathrm{Ni}(111)$, 与 $\mathrm{La} / \mathrm{Ni}(111)$ 相 比, $\mathrm{La}_{2} \mathrm{O}_{3}$ 对 $\mathrm{Ni}$ 的(111) 晶面进行修饰的主要区别是: $\mathrm{La}_{2} \mathrm{O}_{3} / \mathrm{Ni}(111)$ 上 $\mathrm{CH} \rightarrow \mathrm{C}+\mathrm{H}$ 转化速率较低, 且 $\mathrm{Ni}$ 在 $\mathrm{La}_{2} \mathrm{O}_{3}$ 上的分散性良好, 有利于对 $\mathrm{CO}_{2}$ 的吸附, 可以通 过 $\mathrm{CO}_{2}+\mathrm{C} \rightarrow 2 \mathrm{CO}$ 途径来抑制焦炭的产生; 且在 $\mathrm{La}_{2} \mathrm{O}_{3} / \mathrm{Ni}(111)$ 上形成的 $\mathrm{La}_{2} \mathrm{O}_{2} \mathrm{CO}_{3}$ 中间体 $\left(\mathrm{CO}_{2}\left(\mathrm{La}_{2} \mathrm{O}_{2}-\mathrm{O}\right)\right)$ 可有效抑制积碳的产生, 从而提高了催化反应稳定性.
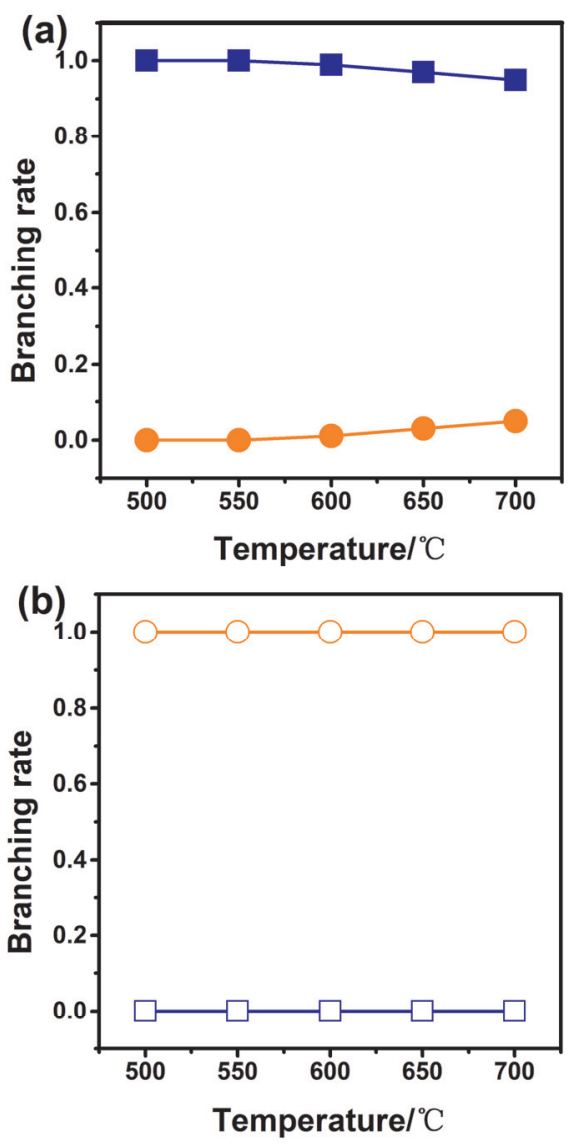

图 8 在 $\mathrm{La} / \mathrm{Ni}(111) 、 \mathrm{La}_{2} \mathrm{O}_{3} / \mathrm{Ni}(111)$ 催化剂上发生 $\mathrm{C}-\mathrm{H}$ 键解离(深蓝 色、、)的速率，以及(a)在 $\mathrm{La} / \mathrm{Ni}(111)$ 上最容易发生的 $\mathrm{P} 2$ 反应(橙色・)、 在 $\mathrm{La}_{2} \mathrm{O}_{3} / \mathrm{Ni}(111)$ 上最容易发生的 $\mathrm{P} 4$ 反应(橙色。)速率 ${ }^{[35]}$

Figure 8 The branching reaction rate of $\mathrm{C}-\mathrm{H}$ dissociation (navy $\mathbf{\bullet}, \mathrm{a}$ ) and the most favorable pathway (a) P2 for La-Ni (orange $\bullet$ ) and (b) P4 for $\mathrm{La}_{2} \mathrm{O}_{3}-\mathrm{Ni}$ (orange $\odot$ ) over $\mathrm{La} / \mathrm{Ni}(111)$ and $\mathrm{La}_{2} \mathrm{O}_{3} / \mathrm{Ni}(111)^{[35]}$

\section{4 总结与展望}

稀土元素独特的外层电子结构特征导致其具有总 体化学性质稳定、元素化学价态可变、多种配位形式、 具有 Lewis 酸性等特点, 且可作为催化助剂调变载体的 形貌、结构、酸碱性等, 因而受到研究者的广泛关注. 本 综述对稀土材料用于(热)催化领域的构效关系做了总 结, 从稀土材料作为载体、Lewis 酸(碱)活性中心、催化 助剂三个不同的方面举例说明了稀土材料电子层结构 与催化反应效果之间的关系, 说明稀土材料在催化领域 
中有着广泛的应用.

但是稀土材料用于催化领域也存在一些问题, 如合 成方法不够环保、催化活性受载体限制较大、作为助剂 的作用机理解释不明确等. 因此需要研究人员的进一步 研究, 以拓展稀土材料在催化领域的应用, 改善我国稀 土资源利用率低的问题, 以期早日实现我国从稀土资源 大国到稀土产业强国的转变.

\section{作者简介}

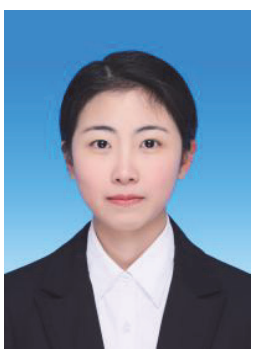

郭金秋, 1997 年出生. 2019 年本科毕业于江苏大学复合材 料与工程专业, 随后加入到南开大学张洪波研究员课题组攻 读硕士学位, 研究方向为催化乙醇生成高碳、高附加值的产 物.

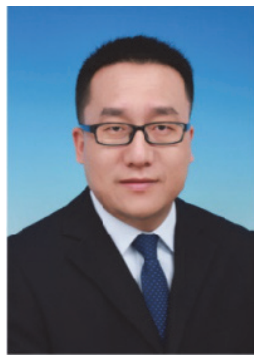

杜亚平, 理学博士, 教授, 博士生导师. 现任天津市稀土 材料与应用重点实验室主任, 南开大学稀土与无机功能材料 研究中心副主任. 2004 年在兰州大学获理学学士学位, 2009 年 在北京大学获博士学位. 近期的研究工作主要集中在新型稀 土纳米结构的研究, 承担和参加国家自然科学基金优青、面上 项目和京津冀协同创新重点项目等研究课题. 近 5 年以通讯 作者或第一作者在 Sci. Adv、A A gew. Chem. Int. Ed.、Adv. Mater、Chem. Soc. Rev. 等刊物发表论文 90 余篇, 获授权专利 3 项. 曾获国家自然科学基金优秀青年基金, 天津市领军人才, 南开大学百名青年学科带头人等, 并担任国际期刊 RSC $A d v$. (RSC) 副主编, 科学通报 Science Bulletin 客座编辑(guest editor), 中国化学快报 Chinese Chemical Letters (Elsevier)青年编委, General Chemistry 杂志编委等.

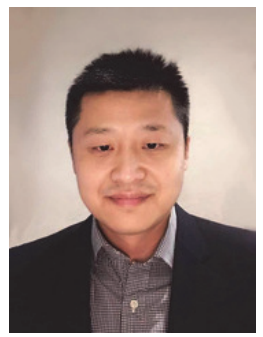

张洪波, 理学博士, 博士生导师, 南开大学材料科学与工 程学院特聘研究员. 2012 年毕业于中国科学院大连化学物理
研究所, 曾先后在美国国家实验室、宾州州立大学以及伊利诺 伊大学香槟分校从事博士后研究工作, 于 2018 年加盟南开大 学, 担任课题组长. 其研究致力于生物质平台小分子的高效 转化, $\mathrm{C}_{1}$ 小分子定向转化, 借助 $\mathrm{X}$ 射线吸收精细结构 (XAFS)、固体核磁共振技术(ssNMR)和傅里叶变换红外吸收光 谱仪(FTIR)等手段对催化剂微观结构进行表征, 并利用化学 反应动力学推测催化反应机理.

\section{致谢}

感谢和志伟、戴菁菁、袁晋、张帅、王妍茹等对这 篇论文提出的修改建议.

\section{References}

[1] Dong, H.; Du, S. R.; Zheng, X. Y.; Lyu, G. M.; Sun, L. D.; Li, L. D.; Zhang, P. Z.; Zhang, C.; Yan, C. H. Chem. Rev. 2015, 115, 10726.

[2] Information Office of the State Council of the People's Republic of China. China Metal Bulletin 2012, 24, 20 (in Chinese). (中华人民共 和国国务院新闻办公室，中国金属通报, 2012, 24, 20.)

[3] Wang, M.; Shen, M.; Jin, X. X.; Tian, J. J.; Li, M. L.; Zhou, Y. J.; Zhang, L. X.; Li, Y. S.; Shi, J. L. ACS Catal. 2019, 9, 4573.

[4] Liang, M. F.; Borjigin, T.; Zhang, Y. H.; Liu, B. H.; Liu, H.; Guo, H. Appl. Catal. B: Environ. 2018, 243, 566.

[5] Zhang, F.; Braun, G. B.; Shi, Y. F.; Sun, X. H.; Reich, N. O.; Zhao, D. Y.; Stucky, G. J. Am. Chem. Soc. 2010, 132, 2850.

[6] Meng, S. Y.; Wang, M. M.; Lü, B. L.; Xue, Q. J.; Yang, Z. W. Acta Chim. Sinica 2019, 77, 1184 (in Chinese). (孟双艳, 王明明, 吕柏 霖, 薛群基, 杨志旺, 化学学报, 2018, 77, 1184.)

[7] Xia, J. L.; Zhao, H. Y.; Pang, W. K.; Yin, Z. Y; Zhou, B.; He, G.; Guo, Z. P.; Du, Y. P. Chem. Sci. 2018, 9, 3421.

[8] Ha, H. W.; Yun, N. J.; Kim, M. H.; Woo, M. H.; Kim, K. Electrochim. Acta 2006, 51, 3297.

[9] Chen, P. L.; Chen, I. W. J. Am. Ceram. Soc. 1993, 76, 1577.

[10] Frey, A. M.; Karmee, S. K.; de Jong, K. P.; Bitter, J. H.; Hanefeld, U. ChemCatChem 2013, 5, 594.

[11] Chen, Z. Y.; Liu, J. W.; Cui, H.; Zhang, L.; Su, C. Y. Acta Chim. Sinica 2019, 77, 242 (in Chinese). (陈之尧, 刘捷威, 崔浩, 张利, 苏成勇, 化学学报, 2019, 77, 242.)

[12] Song, T. Y.; Xu, J. N.; Cheng, G. Z.; Wang, L. Inorganic Chemistry (Third edition), Higher Education Press, Beijing, 2014, p. 816 (in Chinese). (宋天佑, 徐佳宁, 程功榛, 王莉, 无机化学 (第三版), 高等教育出版社, 北京, 2014, p. 816.)

[13] Risse, T.; Shaikhutdinov, S.; Nilius, N.; Sterrer, M.; Freund, H. J. Acc. Chem. Res. 2008, 41, 949.

[14] Mattos, L. V.; Noronha, F. B. J. Catal. 2005, 233, 453.

[15] Summers, J. C.; Ausen, S. A. J. Catal. 1979, 58, 131.

[16] Yao, H. C.; Yao, Y. F. J. Catal. 1984, 86, 254.

[17] Shyu, J. Z.; Otto, K.; Watkins, W. L. H.; Graham, G. W.; Belitz, R. K. J. Catal. 1988, 114, 23.

[18] Kašpar, J.; Fornasiero, P.; Graziani, M. Catal. Today 1999, 50, 285.

[19] Fornasiero, P.; Monte, R. D.; Rao, G. R.; Kašpar, J.; Meriani, S.; Trovarelli, A.; Graziani, M. J. Catal. 1995, 151, 168.

[20] Valsamakis, I.; Flytzani-Stephanopoulos, M. Appl. Catal. B: Environ. 2011, 106, 255.

[21] Al-Sultan, F. S.; Basahel, S. N.; Narasimharao, K. Fuel 2018, 233 , 796.

[22] Zheng, T. T.; He, J. J.; Zhao, Y. K.; Xia, W. Z.; He, J. L. J. Rare Earth 2014, 32, 97.

[23] Korneeva, E. V.; Ivanova, A. S.; Bukhtiyarova, G. A.; Aleksandrov, P. V.; Zaikovskii, V. I.; Prosvirin, I. P.; Noskov, A. S. Kinet. Catal. 2011, 52, 579 .

[24] Mokhnachuk, O. V.; Soloviev, S. O.; Kapran, A. Y. Catal. Today 2007, 119, 145.

[25] Lemonidou, A. A.; Vagia, E. C.; Lercher, J. A. ACS Catal. 2013, 3, 1919.

[26] Li, X. Y.; Li, D.; Tian, H.; Zeng, L.; Zhao, Z. J.; Gong, J. L. Appl. Catal. B: Environ. 2017, 202, 683 .

[27] Sodesawa, T.; Dobashi, A.; Nozaki, F. Kinet. Catal. Lett. 1979, 12, 107.

[28] Luo, J. Z.; Yu, Z. L.; Ng, C. F.; Au, C. T. J. Catal. 2000, 194, 198. 
[29] Singh, S.; Zubenko, D.; Rosen, B. A. ACS Catal. 2016, 6, 4199.

[30] Pawar, V.; Appari, S.; Monder, D. S.; Janardhanan, V. M. Ind. Eng. Chem. Res. 2017, 56, 8448.

[31] Dahdah, E.; Rached, J. A.; Aouad, S.; Gennequin, C.; Tidahy, H. L.; Estephane, J.; Aboukais, A.; Aad, E. A. Int. J. Hydrogen Energy 2017, 48, 12808 .

[32] Liu, H. R.; Wierzbicki, D.; Debek, R.; Motak, M.; Grzybek, T.; Costa, P. D.; Gálvez, M. E. Fuel 2016, 182, 8.

[33] Tsipouriari, V. A.; Verykios, X. E. J. Catal. 1999, 187, 85.

[34] Oemar, U.; Kathiraser, Y.; Mo, L.; Ho, X. K.; Kawi, S. Catal. Sci. Technol. 2016, 6, 1173.

[35] Li, K.; He, F.; Yu, H. M.; Wang, Y.; Wu, Z. J. J. Catal. 2018, 364, 248.

[36] May, Y. A.; Wang, W. W.; Yan, H.; Wei, S.; Jia, C. J. Chin. J. Catal. 2020, 41, 1017.

[37] Zhou, Y.; Chen, A.; Ning, J.; Shen, W. J. Chin. J. Catal. 2020, 41, 928.

[38] Si, R.; Flytzani-Stephanopoulos, M. Angew. Chem., Int. Ed. 2008, $47,2884$.

[39] Fu, Q.; Weber, A.; Flytzani-Stephanopoulos, M. Catal. Lett. 2001, $77,87$.

[40] Zhang, F.; Chan, S. W.; Spanier, J. E.; Apak, E.; Jin, Q.; Robinson, R. D.; Herman, I. P. Appl. Phys. Lett. 2002, 80, 127.

[41] Gatla, S.; Aubert, D.; Agostini, G.; Mathon, O.; Pascarelli, S.; Lunkenbein, T.; Willinger, M. G.; Kaper, H. ACS Catal. 2016, 6, 6151.

[42] Nie, L.; Mei, D. H.; Xiong, H. F.; Peng, B.; Ren, Z. B.; Hernandez, X. I. P.; DeLaRiva, A.; Wang, M.; Engelhard, M. H.; Kovarik, L.; Datye, A. K.; Wang, Y. Science 2017, 358, 1419.

[43] Spezzati, G.; Benavidez, A. D.; DeLaRiva, A. T.; Su, Y. Q.; Hofmann, J. P.; Asahina, S.; Olivier, E. J.; Neethling, J. H.; Miller, J. T.; Datye, A. K.; Hensen, E. J. M. Appl. Catal. B: Environ. 2019, 243, 36.

[44] Liu, L. C.; Corma, A. Chem. Rev. 2018, 118, 4981.

[45] Zhao, S.; Chen, F.; Duan, S. B.; Shao, B.; Li, T. B.; Tang, H. L.; Lin, Q. Q.; Zhang, J. Y.; Li, L.; Huang, J. H.; Bion, N.; Liu, W.; Sun, H.; Wang, A. Q.; Haruta, M.; Qiao, B. T.; Li, J.; Liu, J. Y.; Zhang, T. Nat. Commun. 2019, 10, 3824.

[46] Liu, J. C.; Wang, Y. G.; Li, J. J. Am. Chem. Soc. 2017, 139, 6190.

[47] Jones, J.; Xiong, H. F.; DeLaRiva, A. T.; Peterson, E. J.; Pham, H.; Challa, S. R.; Qi, G. S.; Oh, S.; Wiebenga, M. H.; Hernandez, X. I. P.; Wang, Y.; Datye, A. K. Science 2016, 353, 150.

[48] Fu, Q.; Saltsburg, H.; Flytzani-Stephanopoulos, M. Science 2003, $301,935$.

[49] Dongil, A. B.; Pastor-Perez, L.; Escalona, N.; Sepulveda-Escribano, A. Carbon 2016, 101, 296.

[50] Reina, T. R.; Ivanova, S.; Centeno, M. A.; Odriozola, J. A. Appl. Catal. B: Environ. 2016, 187, 98.

[51] Rodriguez, J. A.; Liu, P.; Stacchiola, D. J.; Senanayake, S. D.; White, M. G.; Chen, J. G. ACS Catal. 2015, 6, 6696.

[52] Micoud, F.; Maillard, F.; Bonnefont, A.; Job, N.; Chatenet, M. Phys. Chem. Chem. Phys. 2010, 12, 1182.
[53] Li, S. W.; Xu, Y.; Chen, Y. F.; Li, W. Z.; Lin, L. L.; Li, M. Z.; Deng, Y. C.; Wang, X. P.; Ge, B. H.; Yang, C.; Yao, S. Y.; Xie, J. L.; Li, Y. W.; Liu, X.; Ma, D. Angew. Chem., Int. Ed. 2017, 56, 10761.

[54] Guo, Y.; Mei, S.; Yuan, K.; Wang, D. J.; Liu, H. C.; Yan, C. H.; Zhang, Y. W. ACS Catal. 2018, 8, 6203.

[55] Xu, J.; Chen, X. Y.; Xu, Y. S.; Du, Y. P.; Yan, C. H. Adv. Mater. 2019, 1806461.

[56] Pudukudy, M.; Yaakob, Z.; Takriff, M. S. Energy Convers. Manage. 2016, 126, 302.

[57] Pudukudy, M.; Yaakob, Z.; Jia, Q. M.; Takriff, M. S. Appl. Surf. Sci. 2019, 467 468, 236.

[58] Lessard, J. D.; Valsamakis, I.; Flytzani-Stephanopoulos, M. Chem. Commun. 2012, 48, 4857.

[59] Akhmedov, V. M.; Al-Khowaiter, S. H. Catal. Rev.-Sci. Eng. 2007, $44,455$.

[60] Jacinto, S.; Ace, M.; Delgado, J. J.; Goguet, A.; Hardacre, C.; Morgan, K. Chem CatChem 2011, 3, 394.

[61] Wang, Z. Q.; Wang, D.; Gong, X. Q. ACS Catal. 2020, 10, 586.

[62] Huang, Z. Q.; Zhang, T. Y.; Chang, C. R.; Li, J. ACS Catal. 2019, 9, 5523.

[63] Sun, J. M.; Zhu, K. K.; Gao, F.; Wang, C. M.; Liu, J.; Peden, C. H. F.; Wang, Y. J. Am. Chem. Soc. 2011, 133, 11096.

[64] Ogo, S.; Onda, A.; Iwasa, Y.; Hara, K.; Fukuoka, A.; Yanagisawa, K. J. Catal. 2012, 296, 24.

[65] Zhang, H. B.; Ibrahim, M. Y. S.; Flaherty, D. W. J. Catal. 2018, 361, 290.

[66] Pang, J. F.; Zheng, M. Y.; He, L.; Li, L.; Pan, X. L.; Wang, A. Q.; Wang, X. D.; Zhang, T. J. Catal. 2016, 344, 184.

[67] Moteki, T.; Flaherty, D. W. ACS Catal. 2016, 6, 4170.

[68] Dai, J. J.; Zhang, H. B. Sci. China Mater. 2019, 62, 1642.

[69] Cota, I.; Ramírez, E.; Medina, F.; Layrac, C.; Tichit, D.; Gérardin, C. J. Mol. Catal. A: Chem. 2016, 412, 101.

[70] Yan, T. T.; Dai, W. L.; Wu, G. J.; Lang, S.; Hunger, M.; Guan, N. J.; Li, L. D. ACS Catal. 2018, 8, 2760.

[71] Wang, Z.; Fongarland, P.; Lu, G. Z.; Essayem, N. J. Catal. 2014, 318,108 .

[72] Meis, N. N. A. H.; Bitter, J. H.; de Jong, K. P. Ind. Eng. Chem. Res. 2010, 49, 1229.

[73] Álvarez, M. G.; Plísková, M.; Segarra, A. M.; Medina, F.; Figueras, F. Appl. Catal. B: Environ. 2012, 113 114, 212.

[74] Liang, Z.; Jiang, D. H.; Fang, G. Q.; Leng, W. H.; Tu, P. X.; Tong, Y. Q.; Liu, L.; Ni, J.; Li, X. N. ChemistrySelect 2019, 4, 4364.

[75] Zhen, K. J.; Li, R. S.; Wang, G. J.; Bi, Y. L.; Kan, Q. B. Catalysis Basics (Third edition), Science Press, Beijing, 2004, p. 239. (in Chinese). (甄开吉, 李荣生, 王国甲，毕颖丽，阚秋斌，催化作用 基础 (第三版), 科学出版社, 北京, 2004, p. 239)

[76] Cunha, A. F.; Mahata, N.; Órfão, J. J. M.; Figueiredo, J. L. Energy Fuels 2009, 23, 4047.

[77] Gao, J.; Hou, Z. Y.; Guo, J. Z.; Zhu, Y. H.; Zheng, X. M. Catal. Today 2008, 131, 278. 